\title{
An Adaptive Fuzzy Controller for Trajectory Tracking of Robot Manipulator
}

\author{
Amol A. Khalate, Gopinathan Leena, Goshaidas Ray \\ Department of Electrical Engineering, Indian Institute of Technology, Kharagpur, India \\ E-mail: gray@ee.iitkgp.ernet.in \\ Received June 24, 2011; revised July 15, 2011; accepted August 20, 2011
}

\begin{abstract}
In this paper, an adaptive fuzzy control algorithm is proposed for trajectory tracking of an n-DOF robot manipulator subjected to parametric uncertainty and it is advantageous compared to the conventional nonlinear saturation controller. The asymptotic stability of the proposed controller has been derived based on Lyapunaov energy function. The design procedure is straightforward due to its simple fuzzy rules and control strategies. The simulation results show that the present control strategy effectively reduces the control effort with negligible chattering in control torque signals in comparison to the existing nonlinear saturation controller.
\end{abstract}

Keywords: Parametric Uncertainty, Lyapunov's Stability, Adaptive Control, Fuzzy Control, Robotic Manipulator

\section{Introduction}

The mathematical model of a dynamic robot manipulator involves highly nonlinearities, strong coupling and uncertain system dynamics [1-4]. To overcome this problem, various adaptive control strategies have been adopted for such uncertain systems including SISO and MIMO systems with guaranteed performance. The parametric uncertainty mainly occurs in robotic manipulator due to the variation in payloads and mass of links. Many efforts have been made in developing control scheme to achieve precise tracking controller of a robot manipulator in presence of parametric uncertainty. The adaptive control and nonlinear saturation control are the two major approaches in dealing the parametric uncertainty of a robot manipulator $[5,6]$.

Fuzzy control has shown a great potential since it is able to handle uncertainty using the programming capability of human control behavior. Many results have been published in area of design and stability of fuzzy control systems $[3,7,8]$. Efforts have been made to improve tracking performance of fuzzy controllers with adaptive algorithms, which are well known as adaptive fuzzy approach [9]. A vast amount of research work on adaptive fuzzy control of nonlinear dynamic systems can be found in [10] and references cited therein.

In this paper, the possibility of replacing the additional control law given in [2] by an equivalent control law based on fuzzy-logic approach has been explored. Subsequently, an equivalent adaptive fuzzy controller is proposed to design a robust control law for trajectory tracking of a two-link robot manipulator with reduced control effort as well as reduced chattering in control force. Initially fuzzy controller is designed from a simple fuzzy IF-THEN rule and then an adaptive control law is adopted to update the parameters of the fuzzy controller during the adaptation procedure. Now, this adaptive fuzzy controller along with simple PD control term is used to construct the final control torque for tracking control of robot manipulators joints.

\section{Dynamic Model and Robust Control Design}

The dynamic equation of $n$-DOF robot manipulator is governed by [11]

$$
M(q) \ddot{q}+C(q, \dot{q}) \dot{q}+g(q)=\tau \text { with } q \in \mathfrak{R}^{n}, \tau \in \mathfrak{R}^{n}
$$

where $M(q)$ is $n \times n$ inertia matrix, the vector $C(q, \dot{q}) \dot{q}$ represents centrifugal and coriolis forces, and $g(q)$ is the vector of gravitational forces and the matrix $N(q, \dot{q})=\dot{M}(q)-2 C(q, \dot{q})$ is skew-symmetric. The above dynamic Equation (1) is linearly parameterizable as 


$$
M(q) \ddot{q}+C(q, \dot{q})+g(q)=Y(q, \dot{q}, \ddot{q}) \theta
$$

where $\theta$ is a constant $p$-dimensional parameter vector and $Y$ is an $n \times p$ matrix of known functions of the generalized coordinates and their higher derivatives.

It is assumed that there exists an unknown bound on parametric uncertainty such that

$$
\|\tilde{\theta}\|=\left\|\theta-\theta_{0}\right\| \leq \rho
$$

For any specific trajectory, $q_{d}, \dot{q}_{d}, \ddot{q}_{d}$ are the desired positions, velocities, accelerations whereas the position and velocity errors are defined as $\tilde{q}=q-q_{d}, \quad \dot{\tilde{q}}=\dot{q}-\dot{q}_{d}$. The adaptive algorithm [1] is very useful for dealing the parametric uncertainties involve in a robot manipulator. The reference velocity and acceleration of the following quantities are defined as

$$
\dot{q}_{r}=\dot{q}_{d}-\Lambda \tilde{q}, \ddot{q}_{r}=\ddot{q}_{d}-\Lambda \dot{\tilde{q}}
$$

and reference velocity error is given as

$$
\sigma=\dot{q}-\dot{q}_{r}=\dot{\tilde{q}}+\Lambda \tilde{q}
$$

The nominal control law is given as

$$
\begin{aligned}
\tau_{0} & =M_{0}(q) \ddot{q}_{r}+C_{0}(q, \dot{q}) \dot{q}_{r}+g_{0}(q)-K \sigma \\
& =Y\left(q, \dot{q}, \dot{q}_{r}, \ddot{q}_{r}\right) \theta_{0}-K \sigma
\end{aligned}
$$

where $\theta_{0}$ represents fixed robot parameter, these parameters are not updated or changed with time. The gain matrices $K$ and $\Lambda$ are positive definite diagonal matrices.

Now, we define the control torque $\tau$ in terms of nominal control vector $\tau_{0}$ as

$$
\begin{aligned}
\tau & =\tau_{0}-Y\left(q, \dot{q}, \dot{q}_{r}, \ddot{q}_{r}\right) u \\
& =Y\left(q, \dot{q}, \dot{q}_{r}, \ddot{q}_{r}\right)\left(\theta_{0}-u\right)-K \sigma
\end{aligned}
$$

where $u$ is an additional control input that will be designed to achieve robustness to parametric uncertainty represented by $\ddot{\theta}$. Substituting the control law (7) into (1) and after simplification we have,

$$
M(q) \sigma+C(q, \dot{q}) \sigma+K \sigma=Y\left(q, \dot{q}, \dot{q}_{r}, \ddot{q}_{r}\right)(\tilde{\theta}-u)
$$

\section{Simple Fuzzy Logic Control}

In [2], the additional input is defined as

$$
u=\left\{\begin{array}{l}
-\rho \frac{Y^{T} \sigma}{\left\|Y^{T} \sigma\right\|} \text { if }\left\|Y^{T} \sigma\right\|>\varepsilon \\
-\rho \frac{Y^{T} \sigma}{\varepsilon} \text { if }\left\|Y^{T} \sigma\right\| \leq \varepsilon
\end{array}\right.
$$

where $\varepsilon>0$ and the upper uncertainty bound is defined as

$$
\left\|\theta-\theta_{0}\right\| \leq \rho=\left(\sum_{i=1}^{p} \rho_{i}^{2}\right)^{1 / 2}
$$

The parameter $\rho$ indicates the measure of uncertainty that may leads to a higher controller gain. To overcome this problem different weights or gains can be assigned to each component of $u(t)$ and it is assumed there exists an upper bound on each parametric uncertainty as

$$
\left|\tilde{\theta}_{i}\right| \leq \rho_{i}, i=1,2, \cdots, p .
$$

The control law (9) can be modified following the discussion in [2] as

$$
u_{i}=\left\{\begin{array}{ll}
-\rho_{i} \frac{\xi_{i}}{\left|\xi_{i}\right|} & \text { if }\left|\xi_{i}\right|>\varepsilon_{i} \\
-\rho_{i} \frac{\xi_{i}}{\varepsilon_{i}} & \text { if }\left|\xi_{i}\right| \leq \varepsilon_{i}
\end{array} ; i=1,2, \cdots, p .\right.
$$

where $\xi_{i}$ denotes the $i^{\text {th }}$ element of the vector $Y^{T} \sigma$, $\varepsilon_{i}$ is $i^{\text {th }}$ element of $\varepsilon$ and $u_{i}$ is $i^{\text {th }}$ element of $u$ (see (9)).

It can be seen if $\varepsilon_{i}$ is chosen very small then the nonlinear saturation control (12) will act as an ON-OFF controller. It is possible to avoid hard switching and also to achieve a better performance if we grade $\xi_{i}$ in number of linguistic terms.

Therefore, we need to construct additional control input $u$ with the help of " $r$ " numbers of IF-THEN fuzzy rules to achieve robustness against the parametric uncertainty. The $j$ th rule is given as:

Rule $j$ :

$$
\text { IF } \xi_{i} \text { is } M_{i}^{j} \text { THEN } u_{i} \text { is } \psi_{i}^{j}
$$

where, $\xi_{i}$ and $M_{i}^{j}, i=1,2, \cdots, p, j=1,2, \cdots, r$ are the premise variables and the fuzzy terms, respectively; $r$ is the number of IF-THEN rule; $\psi_{i}^{j}$ is the singleton fuzzy inference. The crisp value of $u_{i}$ can be obtained after weighted average deffuzzification,

$$
u_{i}=\sum_{j=1}^{r} \psi_{i}^{j} w_{i}^{j}\left(\xi_{i}\right)=\Psi_{i}^{T} W_{i}\left(\xi_{i}\right)
$$

and

$$
\begin{gathered}
w_{i}^{j}\left(\xi_{i}\right)=\frac{\mu_{M_{i}^{j}}\left(\xi_{i}\right)}{\sum_{k=1}^{r}\left(\mu_{M_{i}^{k}}\left(\xi_{i}\right)\right)} ; \\
\sum_{j=1}^{r} w_{i}^{j}\left(\xi_{i}\right)=1, w_{i}^{j}\left(\xi_{i}\right) \in\left[\begin{array}{ll}
0 & 1
\end{array}\right] \text { for all } j \\
W_{i}\left(\xi_{i}\right)=\left[w_{i}^{1} w_{i}^{2}, \cdots, w_{i}^{r}\right]^{T}, \quad \Psi_{i}=\left[\begin{array}{lll}
\psi_{i}^{1} & \psi_{i}^{2}, \cdots, \psi_{i}^{r}
\end{array}\right]^{T} .
\end{gathered}
$$

where $\mu_{M_{i}^{j}}$ is the grade of membership of $\xi_{i}$ in the 
fuzzy set $M_{i}^{j}$ witht $\left|\psi_{i}^{j}\right| \leq \rho_{i}$.

\section{Design of Adaptive Fuzzy Controller}

In this section, we have proposed an adaptive fuzzy logic controller as an additional control input to achieve robustness against the parametric uncertainty. We have summarized the results of proposed work in following theorem.

Theorem: Let the ith element of the additional control input be defined as

$$
u_{i}=\left(\tilde{\Psi}_{i}+\Psi_{o i}\right)^{T} W_{i}
$$

where, $\Psi_{o i}^{T} W_{i}=\left(\Psi_{i}-\tilde{\Psi}_{i}\right)^{T} W_{i}$ is adaptive and $\Psi_{i}^{T} W_{i}$ is fuzzy compensation (14) for $\tilde{\theta}_{i}$ with an adaptive law as

$$
\dot{\tilde{\Psi}}_{i}=\zeta_{i} W_{i}
$$

and the gain matrix $K$ in (8) is chosen such that

$$
K>\gamma_{\max } I \text {, }
$$

where

$$
\begin{aligned}
& \gamma_{\max }=\max \left(\gamma_{i}\right), \\
& \gamma_{i}= \begin{cases}\frac{\left|\xi_{i}\right|\left|\rho_{i}-\Psi_{o i}^{T} W_{i}\right|}{\|\sigma\|^{2}} & \text { if }\|\sigma\|>\varepsilon_{i} \\
\frac{\left|\xi_{i}\right|\left|\rho_{i}-\Psi_{o i}^{T} W_{i}\right|}{\varepsilon_{i}} & \text { if }\|\sigma\| \leq \varepsilon_{i}\end{cases}
\end{aligned}
$$

Then for the trajectory tracking error of a robot manipulator is uniformly ultimately bounded along the trajectory i.e. the tracking errors $\tilde{q}$ and its derivatives $\dot{\tilde{q}}$ converge to zero with application of additional control law $u(t)$ (15) in presence of parametric uncertainities.

Proof: Let us assume that, the parameter vector $\Psi_{o i}$ is selected in an adaptive way so that $\mu_{o i}=\Psi_{o i}^{T} W_{i}$ is the adaptive compensation for $\rho_{i}$.

Let us choose Lyapunov function as

$$
V\left(\sigma, \tilde{\Psi}_{i}\right)=\frac{1}{2} \sigma^{T} M(q) \sigma+\frac{1}{2} \sum_{i=1}^{p} \tilde{\Psi}_{i}^{T} \tilde{\Psi}_{i}
$$

The time derivative of $V$ along the trajectories is

$$
\dot{V}=\sigma^{T} M(q) \dot{\sigma}+\frac{1}{2} \sigma^{T} \dot{M}(q) \sigma+\sum_{i=1}^{p} \tilde{\Psi}_{i}^{T} \dot{\tilde{\Psi}}_{i}
$$
have

Using the property $\sigma^{T}[\dot{M}(q)-2 C(q, \dot{q})] \sigma=0$, we

$$
\dot{V}=-\sigma^{T} K \sigma+\sigma^{T} Y(\tilde{\theta}-u)+\sum_{i=1}^{p} \tilde{\Psi}_{i}^{T} \dot{\tilde{\Psi}}_{i}
$$

Note that $\sigma^{T} Y=\left[\begin{array}{llll}\xi_{1} & \xi_{2} & \cdots & \xi_{\mathrm{p}}\end{array}\right], \tilde{\theta}=\left[\begin{array}{llll}\tilde{\theta}_{1} & \tilde{\theta}_{2} \cdots & \tilde{\theta}_{\mathrm{p}}\end{array}\right]$, (21) can be written as

$$
\dot{V}=-\sigma^{T} K \sigma+\sum_{i=1}^{p} \xi_{i}\left(\tilde{\theta}_{i}-u_{i}\right)+\sum_{i=1}^{p} \tilde{\Psi}_{i}^{T} \dot{\tilde{\Psi}}_{i}
$$

We know that $\left|\tilde{\theta}_{i}\right|=\left|\theta_{i}-\theta_{0 i}\right| \leq \rho_{i}$, and $\xi_{i}$ can be negative also so (23) reduces to

$$
\dot{V} \leq-\sigma^{T} K \sigma+\sum_{i=1}^{p}\left\{\left|\xi_{i}\right|\left(\rho_{i}-u_{i}\right)+\tilde{\Psi}_{i}^{T} \dot{\tilde{\Psi}}_{i}\right\}
$$

Substituting the adaptive fuzzy control from (15)

$$
\begin{aligned}
\dot{V} \leq & -\sigma^{T} K \sigma \\
& +\sum_{i=1}^{p}\left\{\left|\xi_{i}\right|\left(\rho_{i}-\left[\tilde{\Psi}_{i}^{T} W_{i}+\Psi_{o i}^{T} W_{i}\right]\right)+\tilde{\Psi}_{i}^{T} \dot{\Psi}_{i}\right\} \\
\dot{V} \leq & -\sigma^{T} K \sigma \\
& +\sum_{i=1}^{p}\left\{\left|\xi_{i}\right|\left(\rho_{i}-\Psi_{o i}^{T} W_{i}\right)+\left(\tilde{\Psi}_{i}^{T} \dot{\tilde{\Psi}}_{i}-\tilde{\Psi}_{i}^{T} \zeta_{i} W_{i}\right)\right\}
\end{aligned}
$$

Using the adaptive law (16)

$$
\begin{gathered}
\dot{V} \leq-\sigma^{T} K \sigma \\
+\sum_{i=1}^{p}\left\{\left|\xi_{i}\right|\left(\rho_{i}-\Psi_{o i}^{T} W_{i}\right)+\left(\tilde{\Psi}_{i}^{T} \dot{\tilde{\Psi}}_{i}-\tilde{\Psi}_{i}^{T} \dot{\tilde{\Psi}}_{i}\right)\right\} \\
\dot{V} \leq-\sigma^{T} K \sigma+\sum_{i=1}^{p}\left|\xi_{i}\right|\left(\rho_{i}-\Psi_{o i}^{T} W_{i}\right)
\end{gathered}
$$

There exists a small positive scalar $\beta_{i}>0$ satisfying following inequality [12],

$$
\left|\rho_{i}-\Psi_{o i}^{T} W_{i}\right| \leq \beta_{i} \quad i=1,2, \cdots, p .
$$

Similarly, with $\gamma_{i}>0$ we can write,

$$
\left|\rho_{i}-\Psi_{o i}^{T} W_{i}\right| \leq \beta_{i} \leq \gamma_{i} \frac{\|\sigma\|^{2}}{\left|\xi_{i}\right|} \quad i=1,2, \cdots, p .
$$

Substituting (29) in (27),

$$
\dot{V} \leq-\sigma^{T} K \sigma+\sum_{i=1}^{p} \gamma_{i}\|\sigma\|^{2}
$$

Using $\gamma_{\max }$ for $\gamma_{i}$ with $\gamma_{\text {max }}=\max \left(\gamma_{i}\right), i=1,2, \cdots, p$ the above Equation (30) can be written as

$$
\dot{V} \leq-\sigma^{T} K \sigma+\sigma^{T}\left(\gamma_{\max } I\right) \sigma=-\sigma^{T}\left(K-\gamma_{\max } I\right) \sigma
$$

From (17), $K-\lambda_{\max } I>0$, hence $\dot{V} \leq 0$ and the closed-loop system is asymptotically stable and the position and velocity tracking errors $\tilde{q}$ and $\dot{\tilde{q}}$ converge to zero. Moreover, from (29) one can get (18).

The design procedure of adaptive fuzzy controller for robot manipulator can be summarized in following steps:

Step 1: Obtain the parameter vector $\Psi_{i}, i=1,2, \cdots, 6$, according to fuzzy IF-THEN Rules.

Step 2: Using the adaptive law (16) obtain $\tilde{\Psi}_{i}, i=1,2, \cdots, 6$ to compute optimal parameter vector 
$\Psi_{0 i}=\Psi_{i}-\tilde{\Psi}_{i}, \quad i=1,2, \cdots, 6$.

Step 3: Compute the additional control input (15),

$u_{i}=\left(\tilde{\Psi}_{i}+\Psi_{o i}\right)^{T} W_{i}, \quad i=1,2, \cdots, 6$.

Step 4: Obtain $\gamma_{i}$ from (18) and get $\gamma_{\max }=\max \left(\gamma_{i}\right)$ for $i=1,2, \cdots, p$.

Step 5: Choose the gain matrix $K$ such that $K>\gamma_{\max } I$.

Step 6: Obtain the final control torque according to (7) $\tau=Y\left(q, \dot{q}, \dot{q}_{r}, \ddot{q}_{r}\right)\left(\theta_{0}-u\right)-K \sigma$.

\section{Description of Two-Ink Robot Manipulator}

In order to show effectiveness of the proposed controller, computer simulations have been performed with the two-link planar manipulator model [11]. Figure 1 shows this two link planar manipulator with the following parameterizations are considered as

$$
\begin{array}{llrl}
\theta_{1} & =m_{1} l_{c 1}^{2}+m_{2} l_{1}^{2}+I_{1} & & \theta_{2}=m_{2} l_{c 2}^{2}+I_{2} \\
\theta_{3} & =m_{2} l_{1} l_{c 2} & \theta_{4} & =m_{1} l_{c 1} \\
\theta_{5} & =m_{2} l_{1} & \theta_{6} & =m_{2} l_{c 2}
\end{array}
$$

where $m_{1}, m_{2}$ the masses of links 1 and 2 respectively, $l_{1}, l_{2}$ are the lengths of link 1 and 2 respectively and $l_{c 1}, l_{c 2}$ are the lengths where the masses of link 1 and 2 are assumed to be concentrated respectively. Using the above parameters, the matrices $M(q), C(q, \dot{q})$ and the vectors $g(q), \tau$ in (1) are given as

$$
\begin{aligned}
M(q) & =\left[\begin{array}{ll}
\theta_{1}+\theta_{2}+2 \theta_{3} \cos \left(q_{2}\right) & \theta_{2}+\theta_{3} \cos \left(q_{2}\right) \\
\theta_{2}+\theta_{3} \cos \left(q_{2}\right) & \theta_{2}
\end{array}\right] \\
C(q, \dot{q}) & =\left[\begin{array}{ll}
-\theta_{3} \sin \left(q_{2}\right) \dot{q}_{2} & -\theta_{3} \sin \left(q_{2}\right)\left(\dot{q}_{1}+\dot{q}_{2}\right) \\
\theta_{3} \sin \left(q_{2}\right) \dot{q}_{1} & 0
\end{array}\right]
\end{aligned}
$$

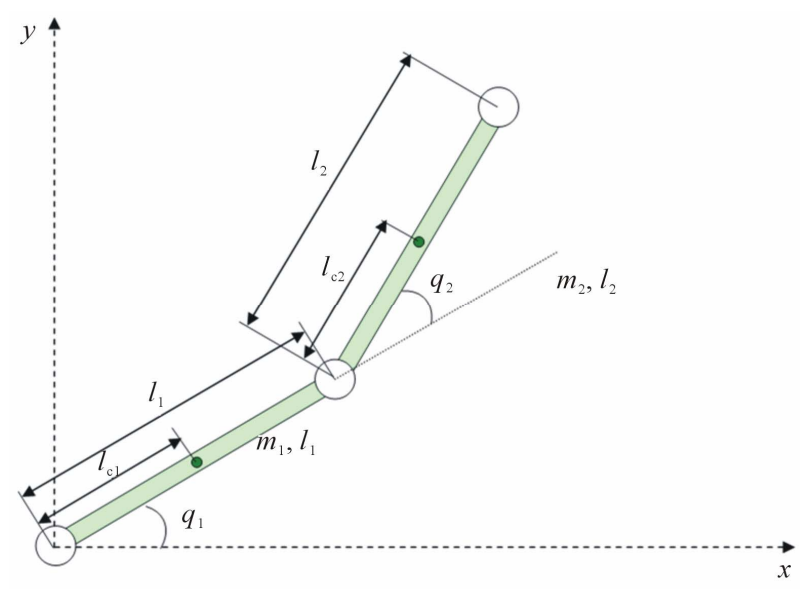

Figure 1. Two-link planar robot.

$$
\begin{gathered}
g(q)=\left[\begin{array}{l}
g\left(\theta_{4}+\theta_{5}\right) \cos \left(q_{1}\right)+g \theta_{6} \cos \left(q_{1}+q_{2}\right) \\
g \theta_{6} \cos \left(q_{1}+q_{2}\right)
\end{array}\right] \\
\tau=Y(q, \dot{q}, \ddot{q}) \theta ; \theta \in \mathfrak{R}^{6}
\end{gathered}
$$

where the component $y_{i j}$ of $Y(q, \dot{q}, \ddot{q})$ are given as

$$
\begin{array}{rlrl}
y_{11}= & \ddot{q}_{1} & y_{12}=\ddot{q}_{1}+\ddot{q}_{2} \\
y_{13}= & \cos \left(q_{2}\right)\left(2 \ddot{q}_{1}+\ddot{q}_{2}\right) & & \\
& -\sin \left(q_{2}\right)\left(\dot{q}_{2}^{2}+2 \dot{q}_{1} \dot{q}_{2}\right) & y_{14}=g \cos \left(q_{1}\right) \\
y_{15}= & g \cos \left(q_{1}\right) & y_{16}=g \cos \left(q_{1}+q_{2}\right) \\
y_{21}=0 & y_{22}=\ddot{q}_{1}+\ddot{q}_{2} \\
y_{23}=\cos \left(q_{2}\right) \ddot{q}_{1}+\sin \left(q_{2}\right) \dot{q}_{1}^{2} & y_{24}=0 \\
y_{25}=0 & y_{26}=g \cos \left(q_{1}+q_{2}\right)
\end{array}
$$

The each component of $Y\left(q, \dot{q}, \dot{q}_{r}, \ddot{q}_{r}\right)$ in (7) is given as

$$
\begin{array}{rlrl}
y_{11}= & \ddot{q}_{r 1} & y_{12}=\ddot{q}_{r 1}+\ddot{q}_{r 2} \\
y_{13}= & \cos \left(q_{2}\right)\left(2 \ddot{q}_{r 1}+\ddot{q}_{r 2}\right) & & \\
& -\sin \left(q_{2}\right)\left(\dot{q}_{2} \dot{q}_{r 2}+2 \dot{q}_{1} \dot{q}_{r 2}\right) & y_{14}=g \cos \left(q_{1}\right) \\
y_{15}= & g \cos \left(q_{1}\right) & y_{16}=g \cos \left(q_{1}+q_{2}\right) \\
y_{21}= & 0 & y_{22}=\ddot{q}_{r 1}+\ddot{q}_{r 2} \\
y_{23}= & \cos \left(q_{2}\right) \ddot{q}_{r 1}+\sin \left(q_{2}\right)\left(\dot{q}_{1} \dot{q}_{r 1}\right) & y_{24}=0 \\
y_{25}=0 & y_{26}=g \cos \left(q_{1}+q_{2}\right)
\end{array}
$$

The unloaded manipulator parameters used in simulation are given in Table 1. Using the values from Table 1 in (31) we can get $\theta_{i}$ as shown in Table 2.

We assume that due to unknown load carried by the robot as part of second link, the parameters $m_{2}, l_{c 2}$ and $I_{2}$ will change to $m_{2}+\Delta m_{2}, l_{c 2}+\Delta l_{c 2}$ and $I_{2}+\Delta I_{2}$, respectively. We will design an adaptive controller that will ensure the asymptotic stability in presence of parameter uncertainties in the intervals

$$
0 \leq \Delta m_{2} \leq 10 ; 0 \leq \Delta l_{c 2} \leq 0.5 ; 0 \leq \Delta I_{2} \leq \frac{15}{12} .
$$

The nominal parameter vector $\theta_{0}$ is shown in Table 3 , is obtained after choosing the mean value for the range of possible $\theta_{i}$. The uncertainty bound for each parameter $\rho_{i}$ is shown in Table 4. The design steps of the proposed adaptive fuzzy controller are discussed below:

\subsection{Controller Design}

Step 1: The parameter vector $\Psi_{i}, i=1,2, \cdots, 6$, is the fuzzy controller consequent can be obtained according to fuzzy IF-THEN rules (13). Each premises variable $\xi_{i}$ is described with help of five fuzzy terms NB, NS, Z, PS, 
Table 1. Parameters of the unloaded arm.

\begin{tabular}{cccccccc}
\hline$m_{1}$ & $m_{2}$ & $l_{1}$ & $l_{2}$ & $l_{c 1}$ & $l_{c 2}$ & $I_{1}$ & $I_{2}$ \\
\hline 10 & 5 & 1 & 1 & 0.5 & 0.5 & $10 / 12$ & $5 / 12$ \\
\hline
\end{tabular}

Table 2. $\theta_{i}$ for the unloaded arm.

\begin{tabular}{rrrrrr}
\hline$\theta_{1}$ & $\theta_{2}$ & $\theta_{3}$ & $\theta_{4}$ & $\theta_{5}$ & $\theta_{6}$ \\
\hline 8.33 & 1.67 & 2.5 & 5 & 5 & 2.5 \\
\hline
\end{tabular}

Table 3. Nominal parameter vector $\boldsymbol{\theta}_{0}$.

\begin{tabular}{cccccc}
\hline$\theta_{01}$ & $\theta_{02}$ & $\theta_{03}$ & $\theta_{04}$ & $\theta_{05}$ & $\theta_{06}$ \\
\hline 13.33 & 8.96 & 8.75 & 5 & 10 & 8.75 \\
\hline
\end{tabular}

Table 4. Uncertainty bounds $\rho_{i}$.

\begin{tabular}{cccccc}
\hline$\rho_{1}$ & $\rho_{2}$ & $\rho_{3}$ & $\rho_{4}$ & $\rho_{5}$ & $\rho_{6}$ \\
\hline 5 & 6.25 & 6.25 & 0 & 5 & 6.25 \\
\hline
\end{tabular}

and PB. Therefore, it requires five rules to describe for each $u_{i}, i=1,2, \cdots, 6$. These fuzzy IF-THEN rules are as follows

1) IF $\xi_{i}$ is NB THEN $u_{i}$ is NB;

2) IF $\xi_{i}$ is NS THEN $u_{i}$ is NS;

3) IF $\xi_{i}$ is Z THEN $u_{i}$ is Z;

4) IF $\xi_{i}$ is PS THEN $u_{i}$ is PS;

5) IF $\xi_{i}$ is PB THEN $u_{i}$ is PB.

These fuzzy rules are generated using the control structure (12) of [2]. The membership functions for input and output variables are shown in Figure 2.

Table 5 shows the parameters of membership functions for input variables and Table 6 shows the parameters of membership functions for output variables. Note that there is no uncertainty in parameter $\theta_{4}$, i.e. $\rho_{4}=0$. Therefore, the component $u_{4}$ of $u$ is zero.

Step 2: $\tilde{\Psi}_{i}, i=1,2, \cdots, 6$ are computed using the adaptive law (16). Now we can get the optimal parameter vector as $\Psi_{0 i}=\Psi_{i}-\tilde{\Psi}_{i}, \quad i=1,2, \cdots, 6$.

Step 3: The additional control input is obtained based on adaptive fuzzy control law (15), $u_{i}=\left(\tilde{\Psi}_{i}+\Psi_{o i}\right)^{T} W_{i}$, $i=1,2, \cdots, 6$.

Step 4: Now we will compute $\gamma_{i}$ using (18) and maximum value of it chosen as $\gamma_{\max }=\max \left(\gamma_{i}\right)$.

Step 5: The gain matrix $K$ is now updated so as to meet the stability condition (17) $K>\lambda_{\max } I$.

Step 6: The final control torque is obtained according to (6) $\tau=Y\left(q, \dot{q}, \dot{q}_{r}, \ddot{q}_{r}\right)\left(\theta_{0}-u\right)-K \sigma$. Note that the proposed control algorithm updates the gain $K$ of PD term unlike the previous algorithm presented in [2].
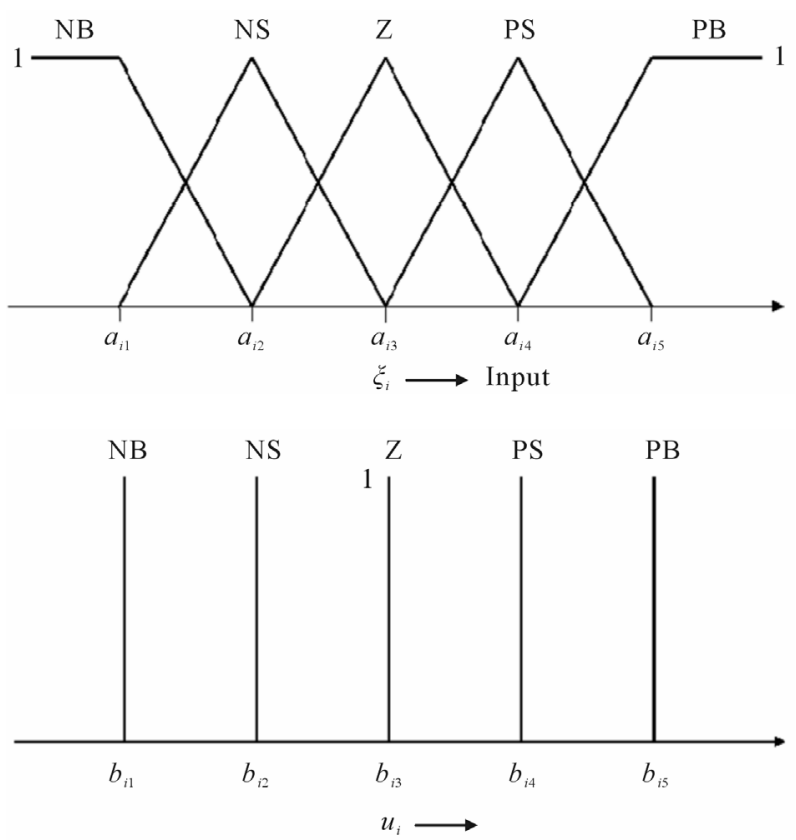

Figure 2. Membership functions for input and output variables.

Table 5. Parameters of membership functions for input variable.

\begin{tabular}{cccccc}
\hline & $a_{i 1}$ & $a_{i 2}$ & $a_{i 3}$ & $a_{i 4}$ & $a_{i 5}$ \\
\hline$\xi_{1}$ & -0.040 & -0.020 & 0.000 & 0.020 & 0.040 \\
$\xi_{2}$ & -0.200 & -0.100 & 0.000 & 0.100 & 0.200 \\
$\xi_{3}$ & -0.150 & -0.075 & 0.000 & 0.075 & 0.150 \\
$\xi_{5}$ & -0.200 & -0.100 & 0.000 & 0.100 & 0.200 \\
$\xi_{6}$ & -0.400 & -0.200 & 0.000 & 0.200 & 0.400 \\
\hline
\end{tabular}

Table 6. Parameters of membership functions for output variable.

\begin{tabular}{cccccc}
\hline & $b_{i 1}$ & $b_{i 2}$ & $b_{i 3}$ & $b_{i 4}$ & $b_{i 5}$ \\
\hline$u_{1}$ & -5.000 & -2.500 & 0.000 & 2.500 & 5.000 \\
$u_{2}$ & -7.290 & -3.645 & 0.000 & 3.645 & 7.290 \\
$u_{3}$ & -6.250 & -3.125 & 0.000 & 3.125 & 6.250 \\
$u_{5}$ & -5.000 & -2.500 & 0.000 & 2.500 & 5.000 \\
$u_{6}$ & -6.250 & -3.125 & 0.000 & 3.125 & 6.250 \\
\hline
\end{tabular}

\subsection{Simulation Results}

Desired trajectories for both the joints are given as

$$
q_{d}=1-\cos (a t), \dot{q}_{d}=a \sin (a t)
$$

where $a=2 \pi / 5$ and simulation results are obtained with a sampling time $\mathrm{T}=0.005 \mathrm{sec}$. Figures 3-6 show 


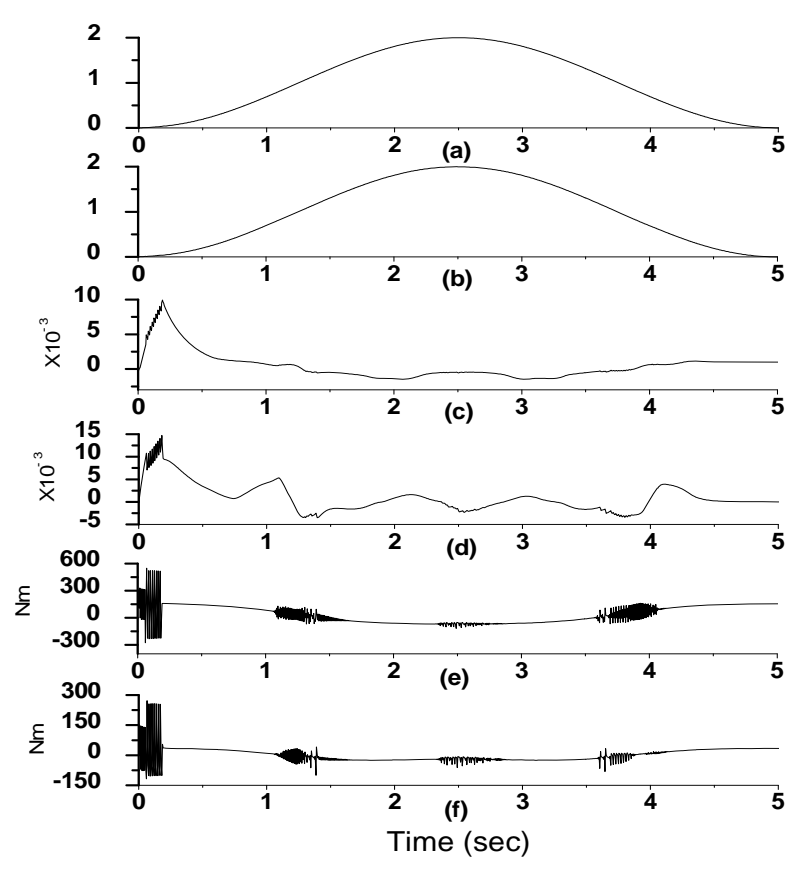

Figure 3. System response based on [2] using controller (12) with $\varepsilon=0.1$.
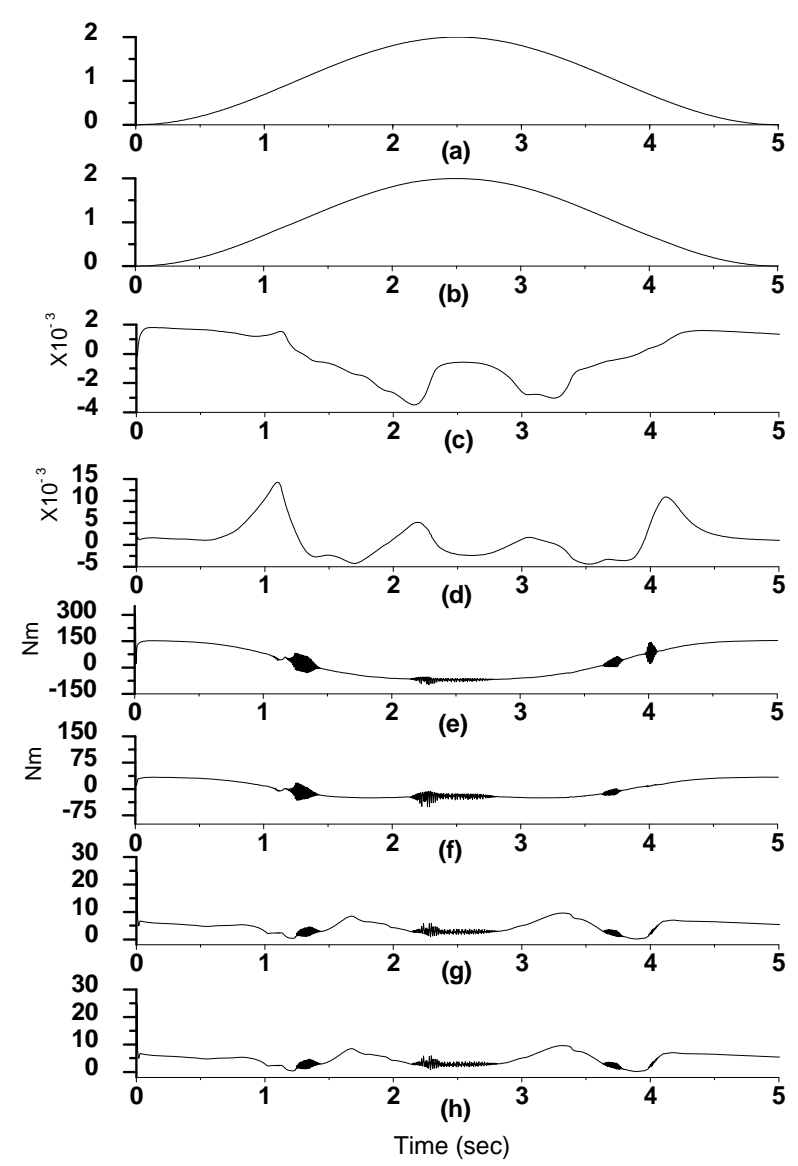

Figure 4. System response based on proposed adaptive fuzzy controller (15) (manipulator is unloaded).

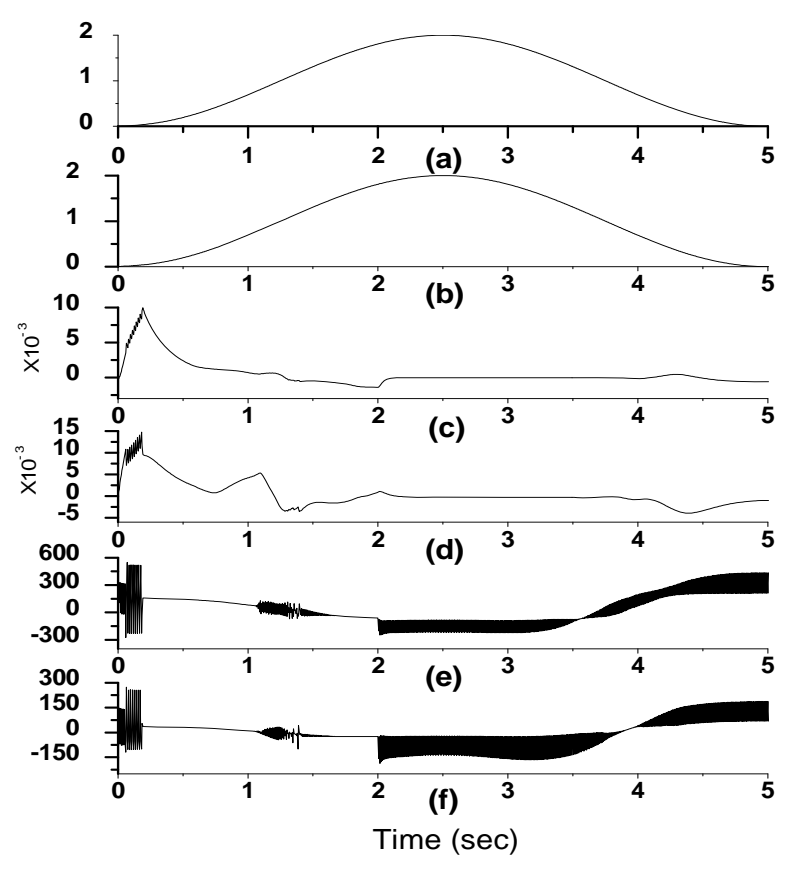

Figure 5. System response based on [2] using controller (12) with $\varepsilon=0.1$ (Manipulator is loaded at time $2 \mathrm{sec}$ ).
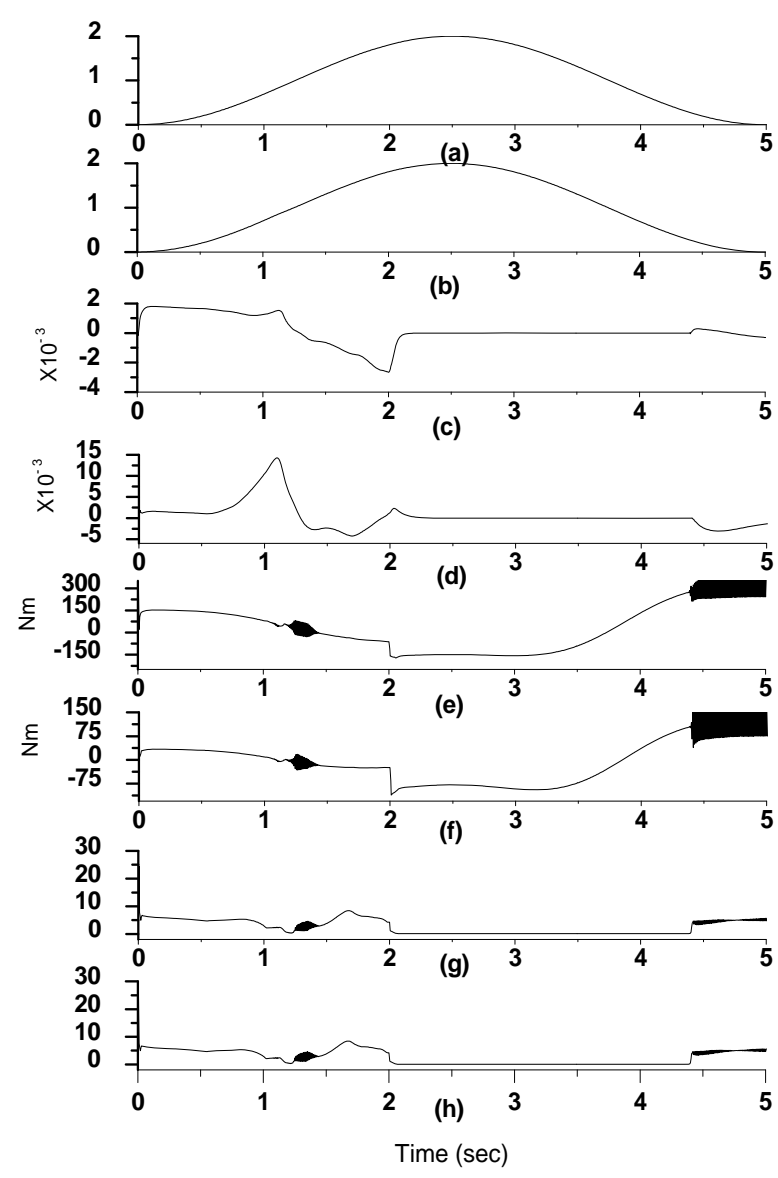

Figure 6. System response based on proposed adaptive fuzzy controller (15) (manipulator is loaded at time 2 sec). 
the system response of a two-link robot manipulator based on nonlinear saturation controller [2] and proposed adaptive fuzzy controller (15) for two cases while the robot manipulator is (i) unloaded (ii) loaded. Results are presented in the following sequences in the figures: joint positions (joint-1 $\left(q_{1}\right)$, joint-2 $\left.\left(q_{2}\right)\right)$, tracking errors $\left(q_{1}\right.$ and $q_{2}$ ), and input torques ( $\tau_{1}$ and $\tau_{2}$ ) respectively. It may be mentioned that the robot manipulator is loaded at time 2 sec. i.e. the second link lifts some mass due to which the parameter of the robot changes as given in (38).

The results of the proposed adaptive fuzzy controller are compared with that of [2] (see (12)) where fixed PD controller gains are considered as $K=\operatorname{diag}(7550)$ and $\Lambda=\operatorname{diag}(4015)$. In both the methods, it is observed that the tracking error responses remain almost the same order and insensitive irrespective of the payload variation. Further it has been observed through simulation studies that the proposed technique effectively alleviates or reduces the chattering effect in control signals. A significant chattering in the control signal is noticed while a robot arm takes a sharp turn under loaded condition. Simulation result shows that the proposed controller effectively reduces the magnitude of input torque or in other words effectively reduces the control effort compared to the method discussed in [2]. It may be further observed from the figures ((see Figures 4(g)-(h) and Figures 6(g)-(h)) how the diagonal elements of gain matrix $K\left(K_{11}, K_{22}\right)$ of PD controller are updated adaptively.

\section{Conclusions}

In this paper, an adaptive fuzzy control law is proposed for trajectory tracking of robot manipulator with a view to reduce the chattering effect in torque control signal. The advantages of fuzzy and adaptive control strategies are combined and subsequently the stability condition of robot manipulator is derived based on Lyapunov theorem. The implementation of proposed controller is very straightforward due to the use of simple fuzzy rules and control strategies. The gain $K$ of PD term is updated with time and hence proposed adaptive control scheme removes the disadvantage of using fixed large gain values in [2]. Simulation results show that the present con- trol scheme is effective in reducing chattering in torque control signal and simultaneously control effort is less in comparison to the results in [2].

\section{References}

[1] J. J. E. Slotine and W. Li, "On the Adaptive Control of Robotic Manipulators," International Journal of Robotics Research, Vol. 6, No. 3, 1987, pp. 49-59. doi: $10.1177 / 027836498700600303$

[2] M. W. Spong, "On the Robust Control of Robot Manipulators," IEEE Transactions on Automatic Control, Vol. 37, No. 11, 1992, pp. 1782-1786. doi:10.1109/9.173151

[3] A. B. Sharkawy, M. M. Othman and A. M. A. Khalil, “A Robust Fuzzy Tracking Control Scheme for Robotic Manipulators with Experimental Verification," Intelligent Control and Automation, Vol. 2, No. 2, 2011, pp. 100-111. doi:10.4236/ica.2011.22012

[4] M. Galicki, "An Adaptive Regulator of Robotic Manipulators in the Task Space," IEEE Transactions on Automatic Control, Vol. 53, No. 4, 2008, pp. 1058-1062. doi:10.1109/TAC.2008.921022

[5] M. W. Spong, S. Hutchinson and M. Vidyasagar, "Robot Modeling and Control," John Wiley \& Sons Inc., New York, 2006.

[6] C. C. Cheah, C. Liu and J. J. E. Soltine, "Adaptive Tracking Control for Robots with Unknown Kinematics and Dynamic Uncertainty," International Journal of Robotics Research, Vol. 25, No. 3, 2006, pp. 283-296. doi:10.1177/0278364906063830

[7] T. H. S. Li and Y. C. Huang, "MIMO Adaptive Fuzzy Terminal Sliding-Mode Controller for Robotic Manipulators," Information Sciences, Vol. 180, No. 23, 2010, pp. 4641-4660. doi:10.1016/j.ins.2010.08.009

[8] Z. Bingul and O. karahan, "A Fuzzy Logic Controller Tuned with PSO for 2 DOF Robot Trajectory Control," Expert Systems with Applications, Vol. 38, No. 1, 2011, pp. 1017-1031. doi:10.1016/j.eswa.2010.07.131

[9] L.-X. Wang, "Stable Adaptive Fuzzy Control of Nonlinear Systems," IEEE Transactions on Fuzzy Systems, Vol. 1, No. 2, 1993, pp. 146-155.

[10] G. Feng, "A Survey on Analysis and Design of ModelBased Fuzzy Control Systems," IEEE Transactions on Fuzzy Systems, Vol. 14, No. 5, 2006, pp. 676-697.

[11] M. W. Spong and M. Vidyasagar, "Robot Dynamics and Control," Wiely, New York, 1989.

[12] L. X. Wang, "A Course in Fuzzy Systems and Control," Prentice-Hall, Englewood Cliffs, 1997. 